\title{
Artículo
}

\section{Encefalitis por anticuerpos anti-NMDAR}

\section{Anti-NMDA antibody encephalitis}

\author{
Dr. Allan Davia Picado Álvarez \\ Médico General graduado de la Universidad de Iberoamérica (UNIBE)
}

Correspondencia con: allanpic93@hotmail.com

\section{Resumen}

La encefalitis por anticuerpos contra los receptores de N-Metil-D-Aspartato (NMDAR) es una patología de reciente descubrimiento, la cual suele iniciar comúnmente con una fase prodrómica "pseudogripal", posteriormente evolucionará con síntomas psiquiátricos, alteración del estado de la conciencia y convulsiones. El diagnóstico suele ser complejo dado la poca incidencia de la misma, además de confundirse con cuadros psiquiátricos y otros tipos de encefalitis. Tiene una presentación clínica grave sin el inicio de un tratamiento precoz, el cual consiste en la administración de corticoesteroides e inmunoglobulinas intravenosas.

\section{Palabras clave}

Encefalitis, autoinmune, neuropsiquiátricos, anticuerpos, receptor N-metil-D-aspartato, convulsiones.

Abstract
Encephalitis due to antibodies against
N-Methyl-D-Aspartate receptors (NMDAR) is a
recently discovered pathology, which usually
begins with a "pseudo-flu" prodromal phase,
later evolving with psychiatric symptoms,
altered state of consciousness and seizures. The
diagnosis is usually complex given the low
incidence of it, in addition to being confused
with other psychiatric conditions and other
types of encephalitis. It has a serious clinical
presentation without timely treatment, which
consists of the administration of corticosteroids
and intravenous immunoglobulins.




\section{Keywords}

Encephalitis, autoimmune, neuropsychiatric, antibodies, N-methyl-D-aspartate receptor, seizures.

\section{Introducción}

Desde su reconocimiento, hace aproximadamente 14 años, este tipo de encefalitis ha evolucionado, ya que al inicio se le consideraba como un extraño síndrome paraneoplásico, hasta constituir en la actualidad una de las principales causas de encefalitis de etiología no viral.

$\mathrm{Su}$ presentación clínica suele ser muy florida, abarcando a través de fases, síntomas psiquiátricos, hasta conducir a una disfunción autonómica e incluso muerte si se atrasan las respectivas terapias, donde el clínico deberá tener en consideración este diagnóstico (1).

Dentro de las principales etiologías, se destacan 2 grupos, donde uno de ellos se relaciona con los tumores ováricos conocidos como teratomas, de ahí que esta enfermedad se conociera como un síndrome paraneoplásico. Además, en grupos etarios de menor edad esta patología se suele relacionar con la exposición a diversos agentes virales, donde se experimenta una reacción de tipo cruzada inmunológica, al igual que con el teratoma (1).

Los anticuerpos son dirigidos en contra de los receptores NMDA, estos constituyen receptores de glutamato que expresan múltiples subunidades que se encuentran en diversos sitios a nivel cerebral, con una importante función en la sinaptogénesis.

La lesión de estos receptores favorece el inicio de cuadros similares a los presentados en enfermedades como la esquizofrenia y la demencia, sin embargo, muchas de las manifestaciones se relacionarán con trastornos del estado de alerta, memoria y sistema autónomo (2). El objetivo del presente artículo es realizar una revisión actualizada sobre esta entidad patológica, para así reconocer y brindar un tratamiento precoz y oportuno, evitando la progresión y las graves complicaciones de la misma.

\section{Método}

Por medio de una revisión bibliográfica, se utilizaron artículos de los últimos cinco años seleccionados de bases de datos como The New England Journal of Medicine (NEJM), The Journal of the American Medical Association (JAMA), Elsevier, PubMed, Scielo, National Library of Medicine, Directory of open access books, Directory of open access journal, utilizando palabras claves como "encefalitis por anticuerpos anti-NMDA", "encefalitis autoinmune", "trastornos neuropsiquiátricos", "anticuerpos", "receptor N-metil-D-aspartato", "convulsiones". Se descargaron un total de 30 artículos de los cuales se descartaron 15 y se utilizaron un total de 15 artículos como referencias bibliograficas, del año 2014 hasta el año 2019.

\section{Epidemiología}

Con respecto a la incidencia de las enfermedades autoinmunes a nivel mundial, esta gira entorno al 5 y $7 \%$, mientras que el 2 y $3 \%$ constituyen la prevalencia de anticuerpos dirigidos contra antígenos neuronales en la población general sana (3).

En el año 2005, 4 mujeres jóvenes con el diagnóstico de teratoma ovárico, presentaron un síndrome, el cual abarcaba un amplio conjunto de síntomas psiquiátricos, asociados a pérdida de la memoria, alteración de la conciencia e incluso hipoventilación. Años más tarde se describió la enfermedad gracias a la identificación de anticuerpos antirreceptor de $\mathrm{N}$-metil-D-aspartato, como un nuevo tipo de encefalitis (4).

Actualmente la incidencia de esta enfermedad es desconocida, gracias en parte al mimetismo que se le puede atribuir con otros síndromes 
similares, por ende, suele ser infradiagnosticada. Suele mencionarse por medio de estudios epidemiológicos que, constituye la primera causa de encefalitis en menores de 30 años y la segunda causa de encefalitis de tipo autoinmune (4).

\section{Receptores nmda}

Son receptores iónicos de glutamato lentos (iGluR) que se dividen en: receptores acoplados a proteínas metabotrópicas G (mGluR) y receptores de glutamato ionotrópicos (iGluR) y conforman los receptores excitadores más significativos del sistema nervioso central (SNC) (5).

Estos receptores expresan las subunidades NR1, NR2 y NR3, donde solo NR1 presenta al menos 8 subunidades distintas, NR2 4 subunidades (A-D) y NR3 únicamente 2 (A-B). Los tetrámeros están compuestos por 2 subtipos de NR1 y 2 subtipos de NR2, también se presentan combinaciones entre los subtipos NR2 y NR3, siendo esto dependiente de la edad del individuo, donde las subunidades NR2 (B y D) suelen presentarse al inicio del desarrollo humano y las subunidades del NR2 (A y C) aparecen durante el crecimiento (2).

La unión activa de la glicina y el glutamato con un receptor NMDAR, provoca la apertura y el ingreso del catión calcio en la célula, dando lugar al inicio de la transducción de señales con la posterior actividad sináptica. Esta es fundamental para la potenciación y actividad sináptica a largo plazo en el hipocampo. Los NMDAR son claves en la modulación de la excitotoxicidad, por lo cual regulan la entrada de cationes (2).

La alteración de la actividad de los NMDAR con una expresión disminuida del NR1 o NR2A, generan cambios característicos del comportamiento, similares a la esquizofrenia, así como múltiples patologías, tales como epilepsia, demencia, enfermedad de Parkinson y Huntington (2).
En cuanto a la distribución cerebral, se expresan de manera generalizada en el cerebro, con una gran concentración en el hipocampo, cerebro anterior y cerebelo. Son fundamentales en la remodelación sináptica y en la sinaptogénesis, con un papel importante en el control conductual, motor, autonómico, respiratorio, así como en la cognición y memoria (3).

\section{Factores de riesgo}

Actualmente, se han agrupado en 2 principales grupos:

Aquellas mujeres con el antecedente de padecer un tumor conocido como teratoma ovárico, con alta incidencia en pacientes afrodescendientes de mayor edad (3).

Se estima que el $20 \%$ de las mujeres afectadas por encefalitis anti NMDAR se encuentran en edades entre los 30 y 35 años, donde se manifiesta como un síndrome paraneoplásico asociado al teratoma ovárico (6). Este tumor suele expresar en su superficie NMDAR, lo cual explica el desarrollo de complejos autoinmunes (2).

En otro gran porcentaje de individuos, especialmente en niños, la encefalitis anti NMDAR se puede presentar por reacción inmunológica cruzada, debido a la exposición a diversos virus, como a el virus del sarampión y paperas, varicela zoster y virus del herpes simple (1).

Otros agentes asociados con la activación de las subunidades del NMDAR son el Mycoplasma pneumoniae, el estreptococo hemolítico del grupo A y el toxoplasma gondii, así como la formación de anticuerpos anti-NMDAR en aquellos pacientes con influenza A /H1N1 (2). 


\section{Patología}

Constituye un tipo de encefalitis de carácter autoinmune, en el que los anticuerpos actúan en contra de los receptores sinápticos de glutamato NMDA (7).

Mediante el estudio histopatológico por medio de biopsias y necropsias cerebrales en aquellos individuos con este tipo de encefalitis, se observaron leves infiltrados de tipo inflamatorio, activación de células de la microglía, depósitos de inmunoglobulinas G (IgG), sin complemento, así como una pérdida discreta de neuronas. Esta es una particular diferencia con los síndromes paraneoplásicos clásicos en los que se produce una degeneración neuronal irreversible a partir de la citotoxicidad dependiente de linfocitos $\mathrm{T}$, mientras como se mencionó previamente, los anticuerpos de la encefalitis anti NMDAR producen cierto grado de disfunción neuronal de carácter reversible con la aplicación de inmunoterapia (8).

Además, se determinó que los anticuerpos son capaces de actuar de forma selectiva, sobre los epítopos NMDAR extracelulares, donde provocan la internalización del receptor, conllevando a una disminución de la sinapsis y relacionándose de manera directa con la presencia de títulos de anticuerpos en LCR. Los receptores tipo 2 de Efrina tipo B (EphB2), los cuales constituyen parte de la familia de los receptores de tirosinaquinasa, que tiene como objetivo la estabilización de los receptores NMDA en la membrana postsináptica, así como la plasticidad sináptica y ciertos mecanismos de potenciación (importantes en los procesos de memoria y aprendizaje), se ven alterados por estos anticuerpos (8).

Es posible identificar en estos sujetos anticuerpos intratecales, producto de la infiltración de las células plasmáticas en el cerebro. Estos anticuerpos son detectables durante y después de la enfermedad, en el periodo de recuperación se observan en bajos niveles, por ende, son un factor importante a la hora de establecer un diagnóstico (8).

\section{Manifestaciones Clinicas}

Las manifestaciones clínicas de esta enfermedad suelen ocurrir en forma de estadios en la mayoría de pacientes, entre las fases que comúnmente se aprecian se encuentran:

\section{Fase Prodrómica}

Aproximadamente, el $70 \%$ de los pacientes presentan síntomas constitucionales que asemejan a un cuadro de infección de vías respiratorias superiores, con malestar general, fiebre, cefalea, el cual no se prolonga por más de dos semanas. Sin el tratamiento oportuno, la patología puede progresar a las siguientes fases (7).

\section{Fase Psicótica}

En esta fase con una duración aproximada de 1 a 3 semanas, predominan los cambios cognitivos y del comportamiento, con deterioro de funciones como atención, memoria, conciencia, así como psicosis con alucinaciones y delirios. La depresión y la paranoia suelen ser síntomas frecuentes en estos pacientes (7).

\section{Fase Neurológica}

Pueden presentarse crisis convulsivas, las cuales son comunes a este nivel de evolución de la enfermedad, donde es usual que sean refractarias a los fármacos anticonvulsivos estándar. Otras manifestaciones que presentan los pacientes antes de llegar a la fase catatónica, son las disfunciones autonómicas, tales como: alteración de la presión arterial, taquicardia, arritmias, hiper o hipotermia, trastornos respiratorios y automatismos motores. Sin un manejo y tratamiento adecuado durante la presencia de manifestaciones autonómicas, la enfermedad puede progresar rápidamente hasta la muerte (6). 


\section{Fase Catatónica}

Conocida como la "fase negativista" ya que incluye un espectro clínico que abarca desde mutismo hasta ausencia de movimientos extraoculares espontáneos o respuesta de cierre. Es usual la presencia de ecolalia, así como ciertas posturas distónicas que preceden a una fase hiperquinética (movimientos coreoatetósicos de las extremidades) (3).

\section{Fase de Recuperación}

Corresponde a la última fase, la cual es gradual e inicia con la desaparición de las disquinesias. Puede tardar entre 2 a 14 meses (3)

\section{Diagnóstico}

El diagnóstico de esta patología, actualmente continúa siendo un desafío para los encargados de las áreas de salud debido a su carácter heterogéneo, donde se identifica en menos del $50 \%$ la etiología específica. Como primer paso para un correcto diagnóstico, es fundamental tener presente que la encefalopatía hace referencia a un estado mental con tendencia a la disminución, donde puede o no existir inflamación del cerebro. Al contrario, durante un episodio de encefalitis existe inflamación cerebral producto de etiologías infecciosas y otras no infecciosas (autoinmunes, vasculares, metabólicas, entre otras) (7).

Entre las manifestaciones clínicas que hacen sospechar del diagnóstico de encefalitis por anticuerpos contra NMDAR se encuentran aquellos pacientes, generalmente mujeres jóvenes con un pródromo similar a un cuadro gripal o a una infección de vías respiratorias superiores, que rápidamente progresan a síntomas psicóticos, así como a un estado de catatonia, con crisis convulsivas e importante disfunción autonómica (3).

El diagnóstico definitivo se logra mediante la obtención de una muestra de LCR, donde se detectará la presencia de anticuerpos anti-NMDAR, sin embargo, no todos los pacientes presentaran títulos séricos de estos, por ende, la ausencia de los mismos no excluye el diagnóstico. La obtención de una biopsia cerebral no suele estar indicada, ya que puede ser normal o tener algunos cambios que resultan inespecíficos tales como la presencia de microglía activa e infiltrados linfocitarios (3).

En el $80 \%$ de los pacientes con la enfermedad, suelen presentar en el LCR, pleocitosis moderada con un $30 \%$ de proteínas y la presencia de bandas oligoclonales en un $60 \%$. Durante las etapas más tempranas de la patología, los análisis del LCR pueden aparentar encontrarse normales, $10 \%$ de los individuos no presentaran anticuerpos detectables en el suero (8).

Suele demostrarse a través de infiltrados perivasculares a nivel del SNC, gran porcentaje de células de tipo B y células plasmáticas, con poca concentración de células $\mathrm{T}$, las cuales se observan en mayor cantidad en la esclerosis múltiple, donde abundan grandes cantidades de macrófagos y pocas cantidades de células B (7).

Recientemente fue aprobado por la FDA (Food and Drug Administration) un ensayo para la detección de anticuerpos IgG contra los receptores NMDA como una prueba cualitativa in vitro para el diagnóstico de esta enfermedad (7).

En cuanto al uso del electroencefalograma (EEG), su utilización es útil en el manejo clínico y como pronóstico en estos pacientes. Presagia una mayor probabilidad de admisión a la unidad de cuidados intensivos (UCI) con empeoramiento clínico, guiando hacia la utilización de medidas más agresivas de tratamiento. La presencia de la onda delta extrema es un patrón visible en esta entidad patológica, sin embargo, no está presente en todos los pacientes con este tipo de encefalitis (9). 
Con respecto al uso de la resonancia magnética (RM) y la fluorodeoxyglucosa tomografía por emisión de positrones (FDG-PET), al menos más de la mitad de las resonancias magnéticas en pacientes con encefalitis anti receptor NMDAR no muestran anomalías. Algunas alteraciones típicas en la RM incluyen la hiperintensidad T2 o en FLAIR, en regiones como: lóbulo temporal medio, hipocampo, lóbulo frontal, ganglios basales, corteza cerebelosa y sustancia blanca subcortical y periventricular. Durante las fases tempranas de la enfermedad, es posible identificar por medio de la FDG-PET datos de hipometabolismo occipito-parietal e hipermetabolismo fronto-temporal (10).

\section{Diagnósticos diferenciales}

Entre los diagnósticos diferenciales a descartar se encuentran: causas infecciosas como las encefalitis virales provocadas por el virus del herpes simple tipo 1 y 2, Epstein Barr virus y varicela zoster, citomegalovirus y enterovirus. Causas psiquiátricas como un episodio psicótico agudo, trastornos afectivos o de conversión, uso de drogas recreacionales como la ketamina, cocaína, anfetaminas y cannabinoides, síndrome serotoninérgico, catatonia letal, encefalitis autoinmune y el síndrome antifosfolipídico (8)

\section{Tratamiento}

Posterior al diagnóstico, se debe iniciar la búsqueda de un teratoma de ovario y apresurar conjuntamente la terapia inmunosupresora. Si se localiza un tumor, la inmunosupresión dependerá de la extirpación del mismo, con un número menor de complicaciones y recaídas a futuro. El manejo se divide principalmente en dos líneas de tratamiento, siendo la primera a base de altas dosis de metilprednisolona, inmunoglobulinas intravenosas y plasmaféresis, mientras que la segunda línea de tratamiento para casos refractarios, es a base de medicamentos como rituximab, ciclofosfamida y metotrexato intratecal (produce disminución de la función linfocitaria a nivel del sistema nervioso central) (7).
Se estima que aproximadamente el 75 a $80 \%$ de los pacientes mejoran con el tratamiento previamente mencionado, sin embargo, el $20 \%$ pueden empeorar e incluso fallecer. Mas de la mitad de los sujetos con solo un mes de tratamiento más resección tumoral (ante existencia del mismo), mostraron una mejoría significativa, con desaparición gradual de los síntomas y disminución de títulos séricos de anticuerpos (7).

Con respecto a las dosis terapéuticas, las pacientes a las que se les extirpó el tumor de ovario, aquellas con un peso mayor de $40 \mathrm{Kg}$ se les brindó 1 gramo de metilprednisolona intravenosa durante 5 días y aquellas con un peso menor de $40 \mathrm{Kg}$ recibieron metilprednisolona $30 \mathrm{mg} / \mathrm{kg}$, 5 dosis mensuales por 6 meses (11).

Ante casos refractarios, donde una terapia de al menos 10 días con fármacos de $1^{\circ}$ línea que no demuestren resultados favorables, ameritará la aplicación de la terapia de segunda línea, con dosis de $375 \mathrm{mg} / \mathrm{m} 2$ semanales por al menos 4 semanas con rituximab, combinado con $375 \mathrm{mg} / \mathrm{m} 2$ de ciclofosfamida y continuado con ciclos mensuales de este.

Se suspende el tratamiento cuando se demuestre una franca mejoría clínica, con disminución cuantitativa de los títulos de anticuerpos. El metotrexato debe aplicarse ante el empeoramiento clínico del paciente que no responde al rituximab con ciclofosfamida (3).

\section{Pronóstico}

En un estudio observacional multiinstitucional efectuado entre el 1 de enero del $2007 \mathrm{y}$ el 1 de enero del 2012 con 577 participantes con un rango etario de entre los 8 meses a 85 años de edad, se analizó la presencia de anticuerpos contra NMDAR en suero y LCR de pacientes con el diagnóstico de encefalitis, de los cuales la totalidad dio positivo para la presencia de estos anticuerpos (12). 
El 81\% de los casos correspondió a mujeres, 211 participantes tenían una edad menor de 18 años y, además, en el $38 \%$ se determinó la presencia de una neoplasia, donde en el 94\% de estos se le atribuyó al teratoma ovárico.

Durante los primeros 24 meses, aproximadamente 394 pacientes obtuvieron resultados favorables con una recuperación favorable y aproximadamente 30 de ellos fallecieron (12).

Se han asociado con peores resultados clínicos, aquellos individuos en los que se les retrasa el inicio de la inmunoterapia. Algunas variantes de mal pronóstico son la alteración de la conciencia, la ausencia de inmunoterapia, edad avanzada y la necesidad de ingreso a la unidad de cuidados intensivos (UCI). Cambios en la resonancia magnética y anormalidades en el LCR no asociaron un valor pronostico significativo (13).

Un estudio realizado con 31 pacientes con el diagnóstico positivo para esta entidad, determinó que aquellos pacientes con alteraciones en el estado de conciencia se asociaban con una mayor tasa de ingreso a la UCI, así como peor pronóstico clínico, ya que se dificultaba el diagnóstico, por ende, una terapia farmacológica precoz (14).

Aquellos pacientes que requirieron terapia adicional con fármacos de $2^{\circ}$ línea, se relacionaron con un mayor empeoramiento de la enfermedad, sin embargo, la aplicación de la misma conllevo a buenos resultados en la salud de estos individuos (15).

\section{Conclusión}

A pesar de la evidencia obtenida a partir del descubrimiento de esta enfermedad en el año 2005, aún no se ha logrado determinar con fortaleza un método diagnóstico eficaz.

Sin embargo, con los avances actuales en la inmunohistoquímica, es probable que prontamente se identifique un método, simple y económico que le facilite al clínico la detección de los agentes autoinmunes involucrados en la patogenia.

Muchos estudios significativos concuerdan en dividir la clínica de esta entidad patológica por medio de fases, ya que la misma suele proliferar con un patrón semiológico característico, navegando desde enfermedades psiquiátricas que asemejan a esquizofrenia o enfermedad de Huntington, hasta episodios de catatonia y alteraciones autonómicas en estadios más avanzados.

Con respecto al manejo, es de relevancia declarar que es una enfermedad tratable, donde las terapias son divididas en escalones o líneas. Existen múltiples fármacos para combatir la enfermedad, sin embargo, lo más importante es la priorización del inicio del tratamiento, ya que, como con otras importantes patologías en medicina el tiempo es fundamental para evitar el avance y mayor repercusión en el individuo. Dentro de las limitantes, se podría establecer que existe un déficit de estudios poblacionales sobre esta enfermedad, así como una escasa clasificación de las muestras en grupos etarios y de género. Es comprensible la dificultad de realizar estudios sobre el tema, producto de la gran diversidad de diagnósticos diferenciales a descartar, debido a una clínica tan abundante presente en estos pacientes. 


\section{Bibliografía}

1. Lynch D, Rattelle A, Dong Y, Roslin K, Gleichman A, Panzer J. Anti-NMDA

Receptor Encephalitis: Clinical Features and Basic Mechanisms. Adv Pharmacology. 2018; 82:235-260.

2. Esposito S, Principi N, Calabresi P, Rigante D. An evolving redefinition of autoimmune encephalitis. Autoimmun Rev.

2019;18(2):155-163.

3. Barquero A. Encefalitis por anticuerpos antirreceptor N-metil-D-aspartato. Acta méd. Costarric. 2016;58(3).

4. Arteche Andrés M, Zugasti Echarte O, de Carlos Errea J, Pérez Rodríguez M, Leyún Pérez de Zabalza R, Azcona Calahorra M. Encefalitis por anticuerpos antirreceptor $\mathrm{N}$-metil-D-aspartato asociada a teratoma ovárico: descripción de un caso e implicaciones anestésicas. Rev Esp

Anestesiol. 2015;62(8):468-471.

5. Huang Q, Xie Y, Hu Z, Tang X. Anti-N-methyl-D-aspartate receptor encephalitis: A review of pathogenic mechanisms, treatment, prognosis. Brain Res. 2020;1727:146549.

6. Weaver M, Griffey R.

Anti-N-Methyl-d-Aspartate Receptor

Encephalitis as an Unusual Cause of Altered Mental Status in the Emergency Department. J Emerg Med.

2016;51(2):136-139.

7. Lazar-Molnar E, Tebo A. Autoimmune NMDA receptor encephalitis. Clin. Chim. Acta. 2015;438:90-97.

8. Guasp M, Dalmau J. Encefalitis por anticuerpos contra el receptor de NMDA. Med Clin. 2018;151(2):71-79.
8. Gillinder L, Warren N, Hartel G, Dionisio S, O'Gorman C. EEG findings in NMDA encephalitis - A systematic review. Seizure. 2019;65:20-24.

9. Bacchi S, Franke K, Wewegama D, Needham E, Patel S, Menon D. Magnetic resonance imaging and positron emission tomography in anti-NMDA receptor encephalitis: A systematic review. J Clin Neurosci.

2018;52:54-59.

10.Chandra S, Ray S, Isaac T, R.Pai A, Krishnareddy H, Dhar D et al. A clinical TRIAD for early suspicion of autoimmune encephalitis as a possibility in patients presenting with progressive cognitive decline. Asian J Psychiatr. 2019;41:5-12.

11.Titulaer M, McCracken L, Gabilondo I, Armangué $\mathrm{T}$, Glaser $\mathrm{C}$, Iizuka $\mathrm{T}$ et al. Treatment and prognostic factors for long-term outcome in patients with anti-NMDA receptor encephalitis: an observational cohort study. Lancet Neurol. 2013;12(2):157-165.

12.Broadley J, Seneviratne U, Beech P, Buzzard $\mathrm{K}$, Butzkueven $\mathrm{H}$, O'Brien $\mathrm{T}$ et al. Prognosticating autoimmune encephalitis: A systematic review. J Autoimmun.

2019;96:24-34.

13. Aungsumart S, Ha A, Apiwattanakul M. Abnormal level of consciousness predicts outcomes of patients with anti-NMDA encephalitis. J Clin Neurosci.

2019;62:184-187.

14. Kong S, Chen Y, Su I, Lin J, Chou I, Chou M et al. Immunotherapy for anti-NMDA receptor encephalitis: Experience from a single center in Taiwan. Pediatr Neonatol. 2019;60(4):417-422. 